\title{
MEASUREMENT OF ROOT LENGTH DENSITY IN INTACT SAMPLES USING X-RADIOGRAPHY AND IMAGE ANALYSIS
}

\author{
Alain Pierret, Chris J MORAn, COLIn B MCLACHLAN AND JOHN M Kirby \\ CSIRO Land \& Water, GPO Box 1666 Canberra ACT 2601, Australia \\ (Accepted May 4, 2000)
}

\begin{abstract}
Measurement of root system attributes is of critical importance to understand and model plant growth. Root length density, the length of roots per unit volume of soil, is one of the important parameters required to understand plant performance. Measuring techniques currently in use to assess this parameter, such as for example core washing, are notoriously imprecise and labour-intensive. Roots and soil being inextricably linked, it is virtually impossible to separate them without loosing a significant amount of the root sample to be measured. This noticeably compromises the accuracy of washing techniques. For this reason, non-invasive measurement approaches are highly desirable. Here, a method based on the combination of X-radiography and image analysis is proposed as a new alternative for the measurement of root length density from intact samples. The successive steps of the method, from sampling to image acquisition are briefly described. A specific measurement algorithm, designed to account for the complex spatial arrangement of the roots within the samples is then presented and discussed in detail.
\end{abstract}

Keywords: root length density, X-radiography, thick sections, projection, linear features.

\section{INTRODUCTION}

Many water and nutrient uptake models currently employed in crop modelling rely on the precise measurement of the root length density, the length of roots per unit volume. The broad categories of methods used to assess root length density can be classified as i) washing methods, where roots are physically extracted from the soil in which they grew and are subsequently measured, ii) mapping methods where root length density is derived from counting the number of root intersections on a pit face, a core face, a polished section or on the sides of minirhizotron tube, and iii) imaging methods, (X-radiography, CAT-scanning, MRI) where roots included within the volume of intact samples are measured directly, without being removed from the soil in which they grew. Washing methods are the most widely used, mainly because of their simplicity. However, their accuracy is questionable since it has been reported that more than $30 \%$ of the roots could be lost during the washing process (Pearson and Jacobs, 1985).

Here, we use X-radiography for detection of plant roots in intact samples. This imaging technique is simple to implement and relatively inexpensive compared to competitive non-invasive techniques such as computer assisted tomography. One of the strengths of this technique is to allow high spatial resolution and image clarity (Wilkins et al., 1996). It is therefore possible to achieve detection of roots down to a radius of $\sim 50 \mu \mathrm{m}$ (Kirby et al., 1998), which potentially improves the accuracy of subsequent root length measurements. In this paper, we present the different steps of this method, from sample preparation to the details of the algorithm used to measure root length density.

\section{MATERIALS AND METHODS}

Undisturbed soil samples are collected by carefully digging cubic samples $\sim 20 \times 20 \times 20 \mathrm{~cm}$ and wrapping them tight in bandages. Blocks collected in the field are deliberately oversized to ensure that a core block $\sim 10 \times 10 \times 10 \mathrm{~cm}$ remains intact after being brought back to the laboratory. Once air-dried, the edges of the initial block are trimmed off and the core block is impregnated with a polyester resin using a vacuum drip method (Moran et al., 1989). After completion of impregnation, 2 sections, one horizontal and one vertical, $\sim 3 \mathrm{~mm}$ thick are cut from the core block using a diamond saw (Fig. 1a). These sections are trimmed down to a size of $7 \times 7 \mathrm{~cm}$ and then thinned to $1 \mathrm{~mm}( \pm 2.5 \%)$ using a polishing machine (Fig. 1b). For practical reasons, primarily 
the degree of complexity and the time involved in sample preparation, it was decided to work with $1 \mathrm{~mm}$ thick soil slices. However, it must be noted that such a thickness, which is about 20 times the radius of the smallest observed features, induces some difficulty for the measurement of root attributes. tend to be bimodal it is related to the relative abundance of pores in the sample. Indeed, due to i) their orientation within the sample, ii) their range of radii which can be more than one order of magnitude, and iii) the presence of abundant cross-overs, roots were found to appear with a very wide range of grey levels in the digitised radiographs.

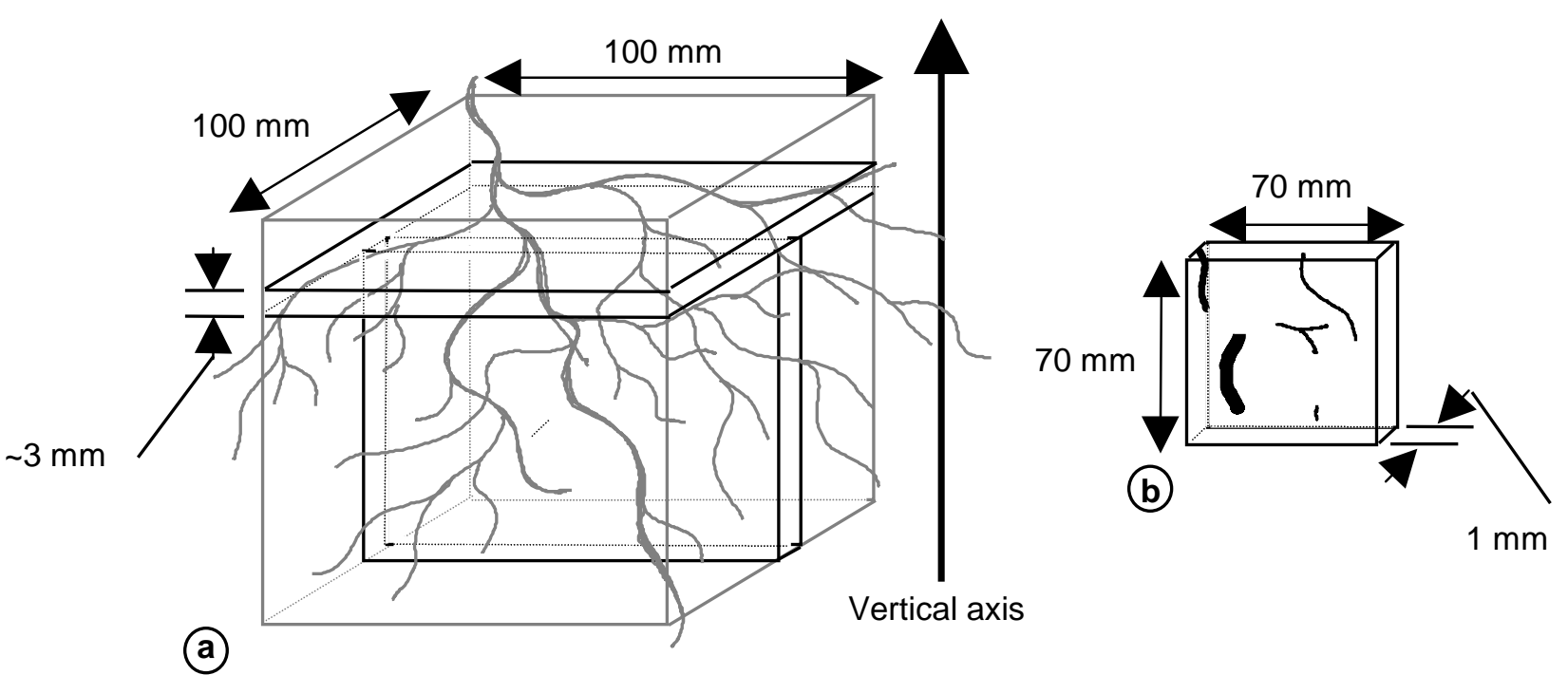

Figure 1. Schematic representation of the sectioning method to obtain vertical and horizontal sections from an impregnated soil sample (a), and actual dimensions of the sections used for subsequent imaging and analysis, (b)(the black fibres represent the sampled fraction of the initial root system).

The imaging technique used in this study, known as X-ray phase contrast imaging (referred to as X-ray PCI), was developed by Wilkins et al. (1996) to detect fine structure in complex and weakly absorbing materials such as biological soft tissues. A conventional microfocus $\mathrm{X}$-ray source is used. There is no need for a highly monochromatic plane-wave radiation nor any sophisticated X-ray optics. The use of a microfocus $\mathrm{X}$-ray source additionally allows high-resolution imaging of X-ray absorbing materials. X-ray PCI was found to provide highly detailed, magnified images of roots in situ together with small features of the soil structure.

On the radiographic film, roots appear as dark fibres, pores in black and gravels in white, whereas soil matrix is grey. Despite clearly identified as dark filaments of variable width with the edges brighter than the axial zone, it was not possible to separate the roots from the soil matrix and the pores on the sole basisof their grey levels. The grey level histograms of the images are mostly unimodal, and when they
As a consequence, it was impossible to automatically segment these images to selectively extract the roots. For that reason, it was decided to manually trace the roots on an acetate sheet fixed over the film, according to a method similar to the one demonstrated by Redon et al. (1998) for fibrous materials. The root tracings and films were digitised using an A4 flatbed document.

\section{MEASUREMENT OF ROOT LENGTH DENSITY}

The root length density, $\mathrm{L}_{\mathrm{V}}$, is the length of roots per unit volume of soil. Despite conceptually simple, $\mathrm{L}_{\mathrm{V}}$ is a variable difficult to estimate accurately (Kücke et al., 1995). Roots observed on the radiographs are projected fibres of small diameter compared to the section thickness. Unbiased stereological methods to measure $\mathrm{L}_{\mathrm{V}}$ from such 2-D projections of fibres have been proposed by Gokhale (1990) and Cruz-Orive and Howard (1991). However, such methods rely on a sufficient number of replicate slices or projection images. Given the time involved in slice preparation 
and root tracing, producing such replicates would unreasonably increase of the time required to process a sample. As a consequence, such methods were not tested here. Instead, a method to assess the minimum and maximum values of $\mathrm{L}_{\mathrm{v}}$ from a single section was designed and implemented. In the case of projected fibres, an estimate of $L_{v}$ based on the length of the skeleton (Russ, 1995) represents the minimum length because of the variable possible orientations of roots within the section. The maximum length can be computed by assuming that every root traverses the section, i.e., one end enters the section at one face and the other exists at the opposite face. With this assumption, knowing the section thickness and the projected length of each root, the angle of incidence of the root with the section plane is computed and the length of the root estimated. Branching and crossing of roots in the projection plane add some complexity to the measurement. This was resolved using an algorithm similar to the one published by Talbot et al. (1996).

The steps involved in this pseudo 3-D reconstruction process are as follows. The root image is skeletonised and a specific search for pixels connected to 3 or more fibres is conducted using a $3 \times 3$ neighbourhood window. Such pixels, defined as nodes, are subsequently removed from the skeleton image and the resulting image of disconnected fibres is stored as a separate file. Concurrently, another file is created to store separately the nodes. A third file is created to store search regions, which are obtained by dilating the nodes. All the fibres, nodes and search regions are labelled. The reconnection process is based on comparing the relative orientations of all fibres included in each search region : fibres with the least different orientations within a given search region are reconnected two by two.

This reconstruction algorithm is sensitive to the size of the search regions as illustrated in Fig. 2. When a cross-over involves two roots which differ in radius (which is the most frequent case), the skeleton of this cross-over looks like the pattern in Fig. 2a. If the small search regions $(1,2)$ from Fig. 2 a are used, the algorithm ends up reconstructing 3 disconnected fibres (Fig. 2b) which, in this case, is a spurious result. The correct reconstruction is achieved by using the large search region (3) from Fig. 2a, so that a cross-over involving only two branches is recognised, as illustrated in Fig. 2c.

Ideally, the size of the search regions should be adjusted for each node, taking into account the initial radii of entering fibres. However, such an approach adds complexity to the algorithm and was therefore not implemented. Alternatively, a single value, at least equal to the radius of the thickest roots imaged, was used. The size of the search regions was thus adjusted to an hexagon of 12 pixels in radius, which, with the resolution used here, corresponds to an actual size of $\sim 400 \mu \mathrm{m}$.

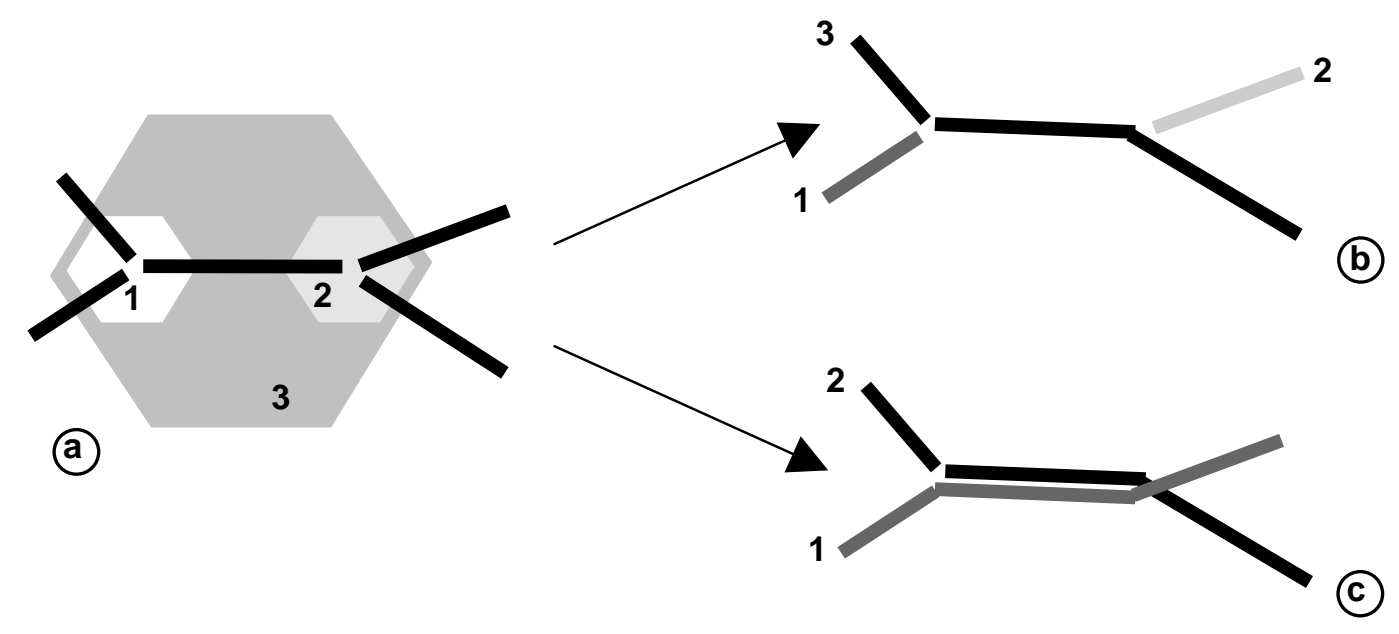

Figure 2. The effect of the size of the search region on the reconstructed system of fibres (see text for details). 
The relevance of this choice was tested by comparing the number of branches and cross-overs as assessed by direct visual inspection of the X-ray images with automatic counts of nodes where 3 fibres meet and nodes where more than 3 fibres meet. These automatic counts were replicated using increasingly larger search regions. The tests were conducted on 12 images. As expected, the comparison showed that if a search region $>12$ pixels in radius was used, the number of branches was underestimated, whereas smaller search regions yielded greater number of branches than those directly observed in the images.

When an odd number fibres enters a search region, the fibre which orientation differs the most from other fibres is assumed to be an individual root and is consequently disconnected from other fibres. This choice was made to ensure that the length density yielded by the algorithm would correspond to the maximum limit for a given geometrical arrangement of fibres. Obviously, it is arguable that this choice may not always correspond to the actual organisation of roots within a sample, and in some instances, such disconnected fibres may in fact be lateral roots. Note that the case just discussed is different from the one illustrated in Fig. 2c where 5 fibres are present in the search region, but 4 only are entering it.

An example of pseudo 3-D reconstruction as used to assess the maximum root length within a sample is presented in Fig. 3 as a perspective view of the reconstructed network of fibres.

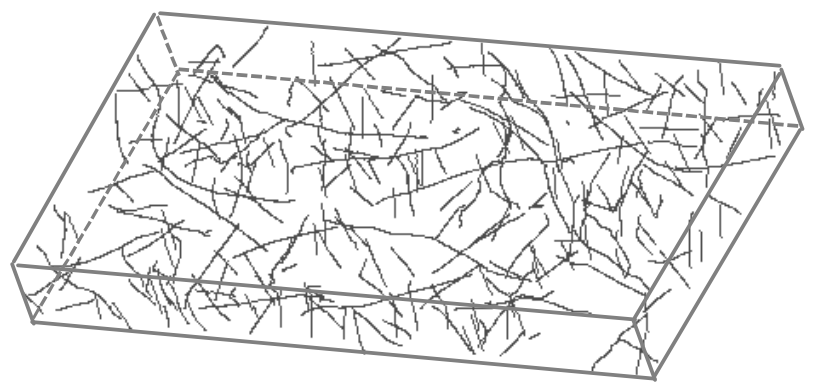

Figure 3. Perspective view of a typical result of the pseudo 3-D reconstruction method.

Both the projection and pseudo 3-D reconstruction methods were used to measure a set of 12 samples corresponding to a field trial sown to wheat (Stewart, 1997). On average, the pseudo 3-D reconstruction yielded estimates $\sim 2$ times larger than the projection method. The estimates of $L_{v}$ yielded by both methods appeared to be $\sim 1$ order of magnitude larger than results from core washing commonly reported in the literature for wheat (Kirby et al., 1998). However, using
X-ray computer tomography, Heeraman et al. (1997) reported figures of similar magnitude for experimentally grown bush beans.

Manual root mapping, followed by stereological measurement of $\mathrm{L}_{\mathrm{v}}$ on the same specimens (Stewart, 1997), yielded estimates of $L_{v}$ comparable to the results obtained using the projection method. However, compared to the manual root mapping used by Stewart (1997), the approach presented in this paper has several advantages. Firstly there is no need for assumptions regarding the degree of anisotropy of the root systems measured. Secondly, although some manual operations are still required, the method presented here is much less time consuming.

\section{CONCLUSIONS}

Recent developments in X-ray imaging techniques allow new approaches to the description of the geometry of intact root systems. In this paper, we combine such new X-ray imaging techniques with appropriate image analysis procedures to assess root length density. Unlike methods currently used, the approach presented here allows the quantification of roots without the need to artificially separate them from the soil in which they grow. This, together with the ability to resolve fine roots has important consequences on the final measurements : the new method was found to yield much larger estimates of root length density than core washing, the currently most widely used and accepted method for assessing this parameter. This new method needs quite evidently to undergo further testing and tuning. However, we propose that because of its potentially high accuracy and its relative simplicity, it is likely to become an important agronomic tool which will participate to improve our understanding of plant performance.

A preliminary report of some of the data was presented at the $X^{\text {th }}$ International Congress for Stereology, Melbourne, Australia, 1-4 November 1999.

\section{ACKNOWLEDGMENTS}

This project was financially supported by the Grain Research Development Corporation. We are grateful to Drs S. Wilkins, A. Stevenson, A. Pogany and D. Gao (CSIRO Manufacturing Science and Technology) for their kind support and constant interest with the project. We should like to acknowledge helpful discussions with Dr John Passioura and Dr John Kirkegaard, CSIRO Plant Industry. 


\section{REFERENCES}

Cruz-Orive LM, Howard V (1991). Estimating the length of a bounded curve in three dimensions using total vertical projections. J Microsc 163:101-13.

Gokhale AM (1990). Unbiased estimation of curve length in 3-D using vertical slices. J Microsc 159:133-41.

Heeraman DA, Hopmans JW, Clausnitzer V (1997). Three dimensional imaging of plant roots in situ with X-ray computer tomography. Plant Soil 189:167-79.

Kirby JM, Pierret A, Moran CJ, Cresswell FJ, Knight JH, Pankhurst CE (1998). CSIRO Land and Water consultancy report 98-58.

Kücke M, Schmid H, Spiess A (1995). A comparison of four methods for measuring roots of field crops in three contrasting soils. Plant Soil 172:63-71.

Moran CJ, McBratney AB, Ringrose-Voase AJ, Chartres CJ (1989). A method for the dehydration and impregnation of clay soil. J Soil Sci 40:569-75.
Pearson CJ, Jacobs BC (1985). Root distribution in space and time in Trifolium subterraneum. Aust J Agric Res 36:601-14.

Redon C, Chermant L, Chermant JL, Coster M (1998). Assessment of fibre orientation in reinforced concrete using Fourier image transform. J Microsc 191:258-65.

Russ JC (1995). The image processing handbook. CRC, Boca Raton, Fla.

Stewart JB (1997). The spatial distribution of plant roots and their interaction with soil structure. $\mathrm{Ph}$. D. Thesis, The University of Sydney.

Talbot H, Jeulin D, Hanton D (1996). Image analysis of insulation mineral fibres. Microsc Microanal Microstrct 7:361-8.

Wilkins SV, Gureyev TE, Gao D, Pogany A, Stevenson AW (1996). Phase-contrast imaging using polychromatic hard X-rays. Nature 384:335-8. 